\title{
Considering the collision probability of Active Debris Removal missions
}

\author{
Aleksander A. Lidtke ${ }^{1}$ \\ University of Southampton, Southampton, SO17 1BJ, UK \\ Hugh G. Lewis ${ }^{2}$, \\ University of Southampton, Southampton, SO17 1BJ, UK \\ Roberto Armellin ${ }^{3}$ \\ Surrey Space Centre, University Of Surrey, Guildford, GU2 7XH, UK \\ Hodei Urrutxua ${ }^{4}$ \\ University of Southampton, Southampton, SO17 1BJ, UK
}

\begin{abstract}
Active Debris Removal (ADR) methods are being developed due to a growing concern about the congestion on-orbit and sustainability of spaceflight. This study examined the probability of an on-orbit collision between an ADR target, whilst being de-orbited, and all the objects in the public catalogue published by the US Strategic Command. Such a collision could have significant effects because the target is likely to be located in a densely populated orbital regime and thus follow-on collisions could take place. Six impulsive and three low-thrust example ADR mission trajectories were screened for conjunctions. Extremely close conjunctions were found to result in as much as $99 \%$ of the total accumulated collision probability. The need to avoid those
\end{abstract}

Email addresses: al11g09@soton.ac.uk (Aleksander A. Lidtke ${ }^{1}$ ),

H.G.Lewis@soton.ac.uk r.armellin@surrey.ac.uk H.Urrutxua@soton.ac.uk (Hodei Urrutxua)

${ }^{1} \mathrm{PhD}$ student, Astronautics Research Group, University of Southampton, UK

${ }^{2}$ Senior Lecturer, Astronautics Research Group, University of Southampton, UK

${ }^{3}$ Senior Lecturer, Surrey Space Centre, University Of Surrey, Guildford, UK

${ }^{4}$ New Frontiers Fellow, Astronautics Research Group, University of Southampton, UK 
conjunctions is highlighted, which raises concerns about ADR methods that do not support collision avoidance. Shortening the removal missions, at an expense of more $\Delta V$ and so cost, will also lower their collision probability by reducing the number of conjunctions that they will experience.

Keywords: Active Debris Removal, conjunction detection, collision probability, risk

\section{Introduction}

Active Debris Removal (ADR) is believed to be necessary in order to stop the collision cascade predicted by Kessler \& Cour-Palais (1978) and preserve access to the vital resource of space (Furuta et al., 2014). Considerable investments are being made world-wide in the development of necessary technologies, and in-orbit validations are likely to materialise in the near future (Zuiani \& Vasile, 2012; Cerf, 2013; Pas et al., 2014; Reed \& Barraclough, 2013). Even business models for ADR companies are being studied (Vance \& Mense, 2013).

Rendezvous and interaction with an uncooperative and unprepared object has never been performed before and, as such, will be challenging. Many different concepts for ADR have been proposed and significant research is being done world-wide in order to reduce the tremendous cost of removing many objects (Chamot et al., 2013; Olympio et al., 2010).

Recent studies have shown that failure of ADR missions may have a detrimental effect on the debris environment (Lewis, 2014). The most severe and damaging outcome of an ADR mission failing would be a catastrophic onorbit collision. This would not only negate the benefit of such an initiative but also undermine its support. Comparison of the collision risk associated with different ADR technologies was carried out by Nock et al. (2013). Work has also been done on reducing the probability of causing orbital collisions through ADR, e.g. fragmenting long electrodynamic tethers (Kim et al., 2010). However, these analyses used long-term collision probability estimation techniques, which do not reflect the trends in collision probabilities that will be seen during operational collision screenings. This paper estimates probabilities of causing orbital collisions that are associated with various proposed ADR approaches using algorithms similar to those used operationally (Flohrer et al., 2009). Only ADR mission trajectories, not technologies, are analysed. However, the type of trajectory often implies sets 
of specific technologies, e.g. low-thrust de-orbiting could be achieved using electric propulsion, drag augmentation or electrodynamic tethers.

First, the method of detecting conjunctions and assessing them in terms of collision probability is described. Then, three example ADR trajectory types, which correspond to different ADR approaches, and three exemplar ADR targets are presented. The trajectories are screened for conjunctions against the public two-line element set (TLE) catalogue and their collision probabilities are compared to one another. A comparison is also made to the collision probabilities that the ADR targets accumulate over a period of time in their current orbits, and conclusions are drawn hence.

\section{Methodology}

This section describes the methods that have been used to quantify collision probabilities of example active debris removal mission trajectories. This methodology is only briefly reviewed here, the used algorithms are described in more detail by Lidtke \& Lewis (2014).

\subsection{Conjunction detection}

A conjunction is defined as an event where the centres of mass of two objects are within a specified distance from one another. Specifically, the time of the closest approach (TCA) was chosen as the conjunction epoch. The collision probability between the two objects may be greater at a different time, if their attitude is accounted for, but this was ignored in this study.

Different distance thresholds in e.g. in-track or cross-track directions can also be used for conjunction detection to account for the fact that the position uncertainties are generally not the same in every direction (Alfano, 1994; Coppola \& Woodburn, 1999) and so conjunctions with the same separation between centres of mass might have different collision probabilities. However, it was decided to account for this by setting the conjunction threshold distance high and equal in every direction, and accepting that certain conjunction geometries may result in very low collision probabilities with such a miss distance. Furthermore, conjunctions between more than two objects were treated as multiple conjunctions between pairs of objects.

The computational time required to identify conjunctions involving all the objects in the public TLE catalogue is significant - 14917 objects had been observed in the 30 days preceding 7 Nov 2013 and their orbits were published 
via Space-Track (2013). This raises the need to implement a number of prefilters, which discard pairs of objects that cannot have a conjunction based on fast to evaluate principles, before the more computationally-intensive rangebased detection. To this end, a set of traditional algorithms, based on the "smart sieve" developed by Rodriguez et al. (2002), was used here.

\subsection{Collision probability estimation}

This study is concerned with the collision probability, $P_{C}$, of ADR missions. A method to establish the uncertainty on the state (position and velocity) of the object is given first. Computation of the collision probability, given the uncertain positions of the objects during the close approach, is described next. Finally, an assumption regarding the physical size of the objects, which is important for $P_{C}$ calculations, is discussed.

\subsubsection{State uncertainty}

Ephemerides of all the objects are known with the accuracy of some Space Surveillance and Tracking (SST) system. Conjunction screenings and assessments, and decisions to mitigate the collision risk, are performed by the operators based on these data. Thus, assuming that the ephemerides are known with the accuracy of the SST system gives an estimate of the collision risk that will be accepted by the operators.

The European Space Agency has defined an accuracy envelope that the European Space Surveillance System (ESSS) shall provide (ESA/ESOC , 2013; Krag et al., 2014). If the system is built according to these requirements, the position of all the objects in orbit will be known with accuracy no worse than 40, 200, and $100 \mathrm{~m}$ in the radial, along-track and cross-track reference frame in the Low Earth orbit (LEO) regime at all times (ESA/ESOC , 2013). These standard deviations can readily be used to construct covariance matrices from which the collision probability can be computed.

Because ESSS will catalogue the LEO objects with the said accuracy at any time, propagation of the covariance is not necessary because, in reality, the position uncertainty may only be less. Lower position uncertainty corresponds to lower collision probabilities, unless an extremely close conjunction is recorded. This behaviour of collision probability with varying magnitude of the orbit uncertainty has been investigated in more detail by Alfano (2005).

\subsubsection{Collision probability estimation}

Every conjunction is analysed in a B-plane frame of reference centred on the primary (the ADR mission target) to compute the collision probability 
of every encounter (Berend, 1999; Alfano, 2007; Chan, 2009). Position covariances of both objects (velocity covariance is ignored) are rotated to the B-plane according to the algorithm given e.g. by Berend (1999). The matrices are added to form a combined covariance matrix $C$, which assumes uncorrelated uncertainties of both states. Rectilinear relative motion and time-invariant position uncertainty are assumed in the vicinity of the TCA, thus allowing the covariance matrix to be projected onto the B-plane and reducing the number of dimensions of the problem from three to two (Berend, 1999; Chan, 2009). McKinley (2002) has shown that even for relative velocities of $0.013 \mathrm{~km} / \mathrm{s}$, the rectilinear relative motion assumption resulted in collision probability estimates to be in the same order of magnitude as when this assumption was relieved. Moreover, Frigm \& Rohrbaugh (2008) found that this assumption held in over $99 \%$ cases for LEO and GEO satellites that they analysed. Thus, this assumption is not expected to affect the results of this study because $P_{C}$ of most conjunctions will not be affected by it.

The position covariance matrix $C$ is then converted into a probability density function (PDF) and integrated inside a circle with radius equal to the combined radii of the two objects (collision radius) and centred on the primary (Berend, 1999; Chan, 2009). This integration yields the probability that both objects' centres of mass will be within the collision radius during the closest approach, i.e. the collision probability $P_{C}$.

The integral of this PDF can be expressed as an infinite series of analytical terms, thus reducing the time required to compute the collision probability for every conjunction (Chan, 2009). However, a sensitivity study revealed that, when the probability density is low, this approach is inaccurate due to floating point truncation errors. Such conjunctions were expected to occur often in this study due to relatively low state uncertainty and large conjunction screening distance. Thus, a direct integration of the PDF, using a two-dimensional Simpson numerical integration scheme with 5000 integration intervals (Press, 2002), was used instead.

\subsubsection{Object physical size}

TLEs come with no information as to the size of the associated objects. Therefore, certain assumptions had to be made to enable the collision radius to be estimated and the $P_{C}$ to be computed.

A database containing the physical radii of objects launched up to 2003 (up to catalogue number 28057), originally compiled by The Aerospace Corporation, was used to allow the collision radius to be computed for some 
Table 1: Radii of the objects according to their types (rocket bodies $(\mathrm{R} / \mathrm{B})$, payloads $(\mathrm{P} / \mathrm{L})$, mission-related objects $(\mathrm{MRO})$, and debris (DEB)) as present in MASTER reference population of 1 May 2009 and discerned in Space-Track's three-line element sets. Details given in text.

\begin{tabular}{rccccc}
\hline Object type & $\mathrm{R} / \mathrm{B}$ & $\mathrm{P} / \mathrm{L}$ & $\mathrm{MRO}$ & $\mathrm{DEB}$ & Other \\
\hline MASTER Object ID & 1 & 2 & 3 & 4 & $1,2,3$, and 4 \\
Average radius (m) & 1.769 & 1.035 & 0.539 & 0.156 & 0.347 \\
Standard deviation (m) & 0.815 & 0.782 & 0.722 & 0.555 & 0.780 \\
\hline
\end{tabular}

conjunctions. For the remainder of the catalogue, statistical data from the MASTER reference population of 1 May 2009, which is a reference population used e.g. in some IADC (Inter-Agency Debris Coordination Committee) studies, were used. This population comprises 19630 objects larger than $10 \mathrm{~cm}$, and associates each object with a hard body radius and a type, i.e. classifies it as a rocket body $(\mathrm{R} / \mathrm{B})$, payload $(\mathrm{P} / \mathrm{L})$, mission-related object (MRO), or debris (DEB). An average radius was computed for all the objects of a given type present in the MASTER reference population. The standard deviation of every group was also found and the results are shown in Table 1.

Some of the MASTER object types can be directly linked to TLEs through three-line element sets that contain information about the type of certain objects in their common name fields. Because the three-line element set catalogue does not distinguish mission-related objects, the data for this type of object were not directly utilised. Moreover, three-line element sets of some objects do not classify the objects as payloads, rocket bodies or debris. For these objects, the average size of the entire MASTER 2009 (all four types of objects) population was used.

When an object was not present in the database of radii and $P_{C}$ had to be computed for it, the average radius from Table 1 for the given object type was used. There are many small objects in the MASTER population classified as debris or mission related objects, which means the average radius of those groups of objects is low. However, in both groups, objects much larger than the mean exist, which gives rise to large standard deviations of the samples. This signifies that using one radius for every group of objects is a simplification. It was, however, necessary to retain objects in the analysis to keep the number of conjunctions in orbit similar to what could be achieved with a complete database of object sizes. 
The effect of assuming a radius for many of the objects was investigated by finding all the conjunctions with the public TLE catalogue, closer than $20.0 \mathrm{~km}$, over one year for Envisat and Delta $1 \mathrm{R} / \mathrm{B}$ (catalogue numbers 27386 and 00862 , respectively). These two objects are located in different orbital regimes (LEO and GTO) and so take part in conjunctions with different secondary objects; their conjunctions also typically have different geometries. When the assumed radius of the objects was varied by \pm one standard deviation around the MASTER 2009 average, the collision probabilities accumulated by Delta $1 \mathrm{R} / \mathrm{B}$ and Envisat changed by no more than $+23.9 \%$ and $-3.2 \%$. Thus, assuming a radius for certain objects will not affect the found collision probabilities more than other assumptions, which will all be discussed before conclusions from this study are drawn.

\subsubsection{Verification results}

The developed conjunction detection and $P_{C}$ estimation framework was verified by Lidtke \& Lewis (2014). All the conjunctions of Envisat with the entire public TLE catalogue closer than $20 \mathrm{~km}$ were found between 23 Oct 2013 and 23 Oct 2014. The obtained accumulated collision probability was compared to the values obtained with Systems Toolkit's Conjunction Analysis Tool (STK CAT). Due to STK CAT limitations, it was not possible to assign different radii to objects not present in the physical radii's database based on their type. Therefore, for verification purposes only, all the objects not present in the database were given a radius of $1.0 \mathrm{~m}$. The achieved error in the final accumulated collision probability was $11.6 \%$. This discrepancy can be attributed to different state vectors, and hence also conjunction geometries, returned by the two SGP4 propagators with the same TLEs at the same epochs. Because STK's source code is proprietary, it was impossible to investigate the exact origin of these differences. Uncertain propagation accuracy would be an issue from an operations point of view. However, this study compared the $P_{C}$ accumulated by different ADR mission trajectories. Because all of the trajectories were studied using the same propagator, this comparison remained meaningful in spite of the uncertainty in the propagator results.

\section{Study setup}

Three example active debris removal trajectory types and three targets have been chosen for this study. These do not represent any particular so- 
lutions but have been selected to be different enough to represent as wide a spectrum of potential ADR implementations as possible.

General assumptions, applicable to all the investigated ADR trajectories, are given first. Then, the assumptions applicable to each one individually will be discussed in turn.

\subsection{General assumptions}

Three exemplar ADR targets were selected, and removal of each using the investigated trajectory types was simulated. All of these targets are rocket bodies, which have the potential to take part in catastrophic collisions and hence are suggested for ADR, e.g. by McKnight et al. (2014) or Ash et al. (1993). Namely, they are upper stages of Zenit-2, Tsyklon-3, and Kosmos$3 \mathrm{M}$.

Every ADR trajectory targeting a given object started from the state vector given in Table 2 . Infinitely more trajectories de-orbiting these objects could be devised by changing e.g. the initial epoch. However, the states from Table 2 serve as examples, and using the same initial state vector for all trajectory types allows differences between them to be clearly shown.

Table 2 also shows the orbital regime, in which the given object is located (altitude, inclination and eccentricity), as well as its physical properties, which were used during propagation and to compute the $P_{C}$. These properties were obtained either from dedicated references for Zenit-2 (Zak, A., 2013) and Tsyklon-3 (Zak, A., 2015), or from the MASTER 2009 population introduced in section 2.2.3.

No architecture-specific mass or size additions have been investigated because this study was aimed at investigating general trends exhibited by various groups of ADR concepts rather than specific solutions. Similarly, all phases of the missions that precede the de-orbiting have been ignored because this study was not aimed at investigating various debris capture mechanisms, guidance, navigation and control algorithms, sensors etc.

\subsection{Assumptions specific to individual trajectory types}

Three example trajectory types, which can be associated with particular ADR technologies, have been chosen for the study. All of them shall now be described in turn. 
Table 2: Initial state vectors, orbital regimes, and physical properties of Zenit-2, Tsyklon-3, and Kosmos-3M. Positions and velocities are the Cartesian components in the J2000 frame of reference.

\begin{tabular}{r|r|r|r} 
& \multicolumn{1}{|c|}{ Zenit-2 } & Tsyklon-3 & Kosmos-3M \\
\hline Epoch (UTC) & 1 Jan 2020 0:0:0.0 & 2 Jun 2015 0:0:0.0 & 1 Jan 2017 0:0:0.0 \\
$X(\mathrm{~km})$ & 7183.000 & 6997.000 & 7019.860 \\
$Y(\mathrm{~km})$ & 0.000 & 0.000 & 0.000 \\
$Z(\mathrm{~km})$ & 0.000 & 0.000 & 0.000 \\
$V_{X}\left(\mathrm{~km} \mathrm{sec}^{-1}\right)$ & 0.000 & 0.000 & 0.000 \\
$V_{Y}\left(\mathrm{~km} \mathrm{sec}^{-1}\right)$ & -1.076 & 0.986 & -1.051 \\
$V_{Z}\left(\mathrm{~km} \mathrm{sec}^{-1}\right)$ & 7.375 & 7.492 & 7.475 \\
\hline Inclination $(\mathrm{deg})$ & 98.3 & 82.5 & 98.0 \\
Altitude (km) & 805 & 619 & 667 \\
Eccentricity (-) & 0.0011 & 0.0024 & 0.0027 \\
\hline Mass (kg) & 8900 & 1407 & 1434 \\
Radius (m) & 6.377 & 2.082 & 3.447
\end{tabular}

\subsubsection{Impulsive}

Many ADR solutions that are currently being investigated are based on chemical propulsion and most of them are being designed to remove five objects per year (Cerf, 2013; Pas et al., 2014; Braun et al., 2013). In order to reduce the overall cost of ADR, as few launches as possible are sought. This typically results in trajectories that visit many objects rather than dedicated missions to remove individual targets. In order to reduce the amount of fuel on-board, which is also associated with cost, long coast phases are present in such trajectories. During these phases, the natural gravity perturbations change the relative right ascension between the ADR vehicle and the target without any fuel expense (Cerf, 2013).

The main advantage of chemical propulsion for ADR applications is the capability to target the re-entry into the region of South Pacific Ocean Uninhabited Area (SPOUA) spanning from 29 to 60 degrees south latitude, and 85 to 175 degrees west longitude (Martinez et al., 2014). This allows the casualty risk on the ground to be reduced and maintained at acceptable levels. It was decided that this targeted re-entry should therefore be included in this trajectory.

The initial state vector of Zenit-2 did not allow an immediate de-orbit manoeuvre that ensured re-entry over SPOUA. Hence a drift of almost 12 days was conducted, which ensured that the Zenit-2 R/B's ground track crossed 


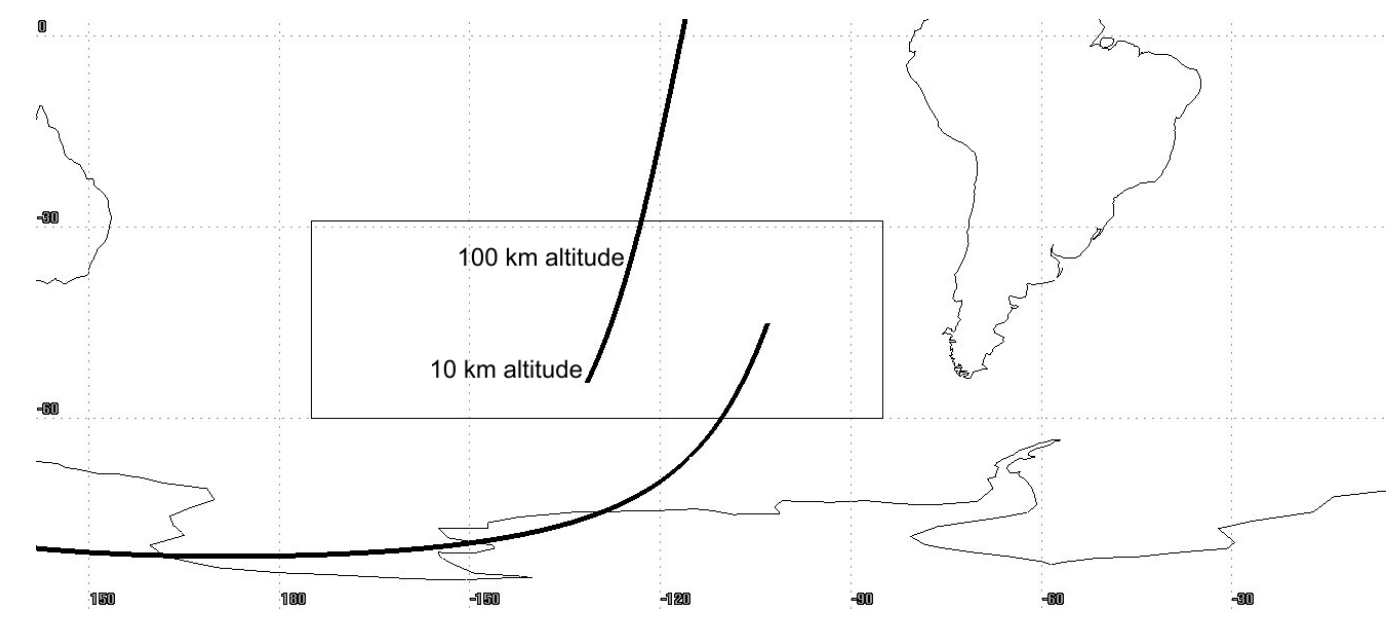

Figure 1: The ground track of the final revolution and re-entry of the impulsive trajectory targeting Zenit-2. South Pacific Ocean Uninhabited Area marked with a black rectangle.

SPOUA close to its centre and an impulsive de-orbit manoeuvre along the orbital velocity direction was conducted then. The epoch and magnitude of the burn were chosen so as to ensure that the entire part of the trajectory below $100 \mathrm{~km}$ altitude was above SPOUA and that the perigee altitude of the last revolution was $0 \mathrm{~km}$ (Martinez et al., 2014). The ground track of the final revolution of the developed trajectory is shown in Fig. 1. This trajectory could be optimised to reduce the total $\Delta V$ but the study was aimed at investigating the general trends in the collision probability rather than finding the best possible trajectories, so the optimisation was not performed.

Coast phases of the same duration and a de-orbit manoeuvre, which met the same requirements (perigee radius, re-entry over SPOUA) were also used in the trajectories de-orbiting Tsyklon-3 and Kosmos-3M. This ensured that all the trajectories were similar, which should result in a comparable number of conjunctions. In principle, the coast duration will be dictated by the epoch when the ADR mission begins and the available fuel. However, making sure that all the trajectories are of similar duration (tailored to the longest trajectory, i.e. Zenit-2) enables a meaningful comparison between them otherwise some trajectories might experience no conjunctions, if the initial epoch happens to enable an immediate re-entry over SPOUA, for example. 


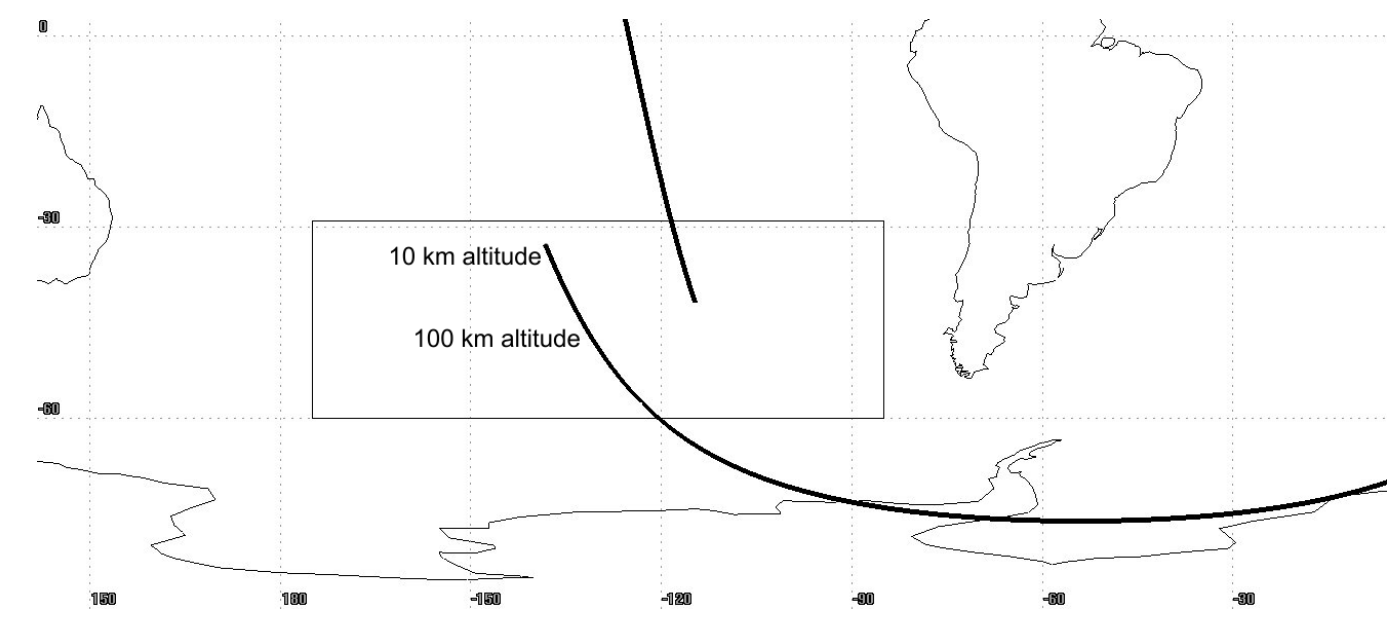

Figure 2: The ground track of the final revolution of the impulsive trajectory targeting Zenit-2 that does not employ natural right ascension drift but uses a manoeuvre to take the trajectory over SPOUA for re-entry. South Pacific Ocean Uninhabited Area marked with a black rectangle.

\subsubsection{Impulsive without extensive drift}

If a single debris object is targeted by an ADR mission, sufficient fuel may be available to avoid long coast phases. This should reduce the collision risk because the mission will be shorter, while requiring larger active right ascension changes and hence larger fuel mass. This may be desired and intended or spare fuel may simply be available in the spacecraft close to the end of its life.

This trajectory is conceptually similar to the impulsive one described in section 3.2.1 because a targeted de-orbit into SPOUA is performed. However, rather than coasting until the trajectory passes over the centre of the target re-entry area, an inclination change manoeuvre is performed to take the spacecraft directly over SPOUA after the first three orbital revolutions. The inclination change is performed together with a manoeuvre along the velocity direction that reduces the perigee altitude to $0 \mathrm{~km}$. As in the impulsive case, the same manoeuvres were performed for all three targets. A part of the ground track of this trajectory for Zenit-2 is shown in Fig. 2. 


\subsubsection{Low-thrust}

Low-thrust trajectories that employ thrust of $25 \mathrm{mN}$ in the anti-tangential direction were also examined. In these trajectories, the altitudes of Zenit-2 and Kosmos-3M were reduced to approximately 550 and $300 \mathrm{~km}$, respectively. The altitude of Tsyklon-3 was lowered to $450 \mathrm{~km}$ using a thrust of $30 \mathrm{mN}$ to provide a low-thrust trajectory that has a shorter duration than the others. An example of the evolution of the instantaneous radius (computed at 1 day intervals) for a low-thrust trajectory targeting Zenit-2 is shown in Fig. 3.

In principle, further lowering of the altitudes is possible at an expense of longer missions and more propellant, which would also increase the cost. The cost would increase even more if altitudes were lowered further because of the increased aerodynamic torques, which would require larger attitude control effort to maintain the thrust alignment. However, because these altitudes ensure re-entry within 25 years from the end of the ADR mission, they were considered sufficient. Performing a controlled re-entry is desirable but targeting a re-entry area is not possible with low-thrust trajectories. However, low-thrust re-entry strategies, which lower the on-ground casualty risk below the risk associated with natural re-entry of the object, have been found to be feasible (Gaudel et al., 2014).

In the scope of ADR architectures, a low-thrust trajectory is often associated with e.g. electrodynamic tethers or drag augmentation devices, and not only traditional low-thrust engines. Such de-orbiting technologies offer considerable cost savings to ADR in general and are thus often favoured. This is mainly because removal of many objects per year is deemed necessary and lowering the cost of de-orbiting individual objects appears to make such an approach to ADR economically feasible.

\section{Results}

For each of the three trajectories de-orbiting or lowering the altitudes of the targets given in section 3, conjunctions were found and assessed according to the algorithm outlined in section 2. Because no catalogue of Earthorbiting objects at the epochs of the ADR trajectories is available, every trajectory was screened against pubic TLE catalogue snapshots obtained on 23 Oct 2013 and 7 Nov 2014. Using different snapshots changed the conjunctions that every trajectory experienced and hence enlarged the sample, on which the conclusions of this work are based. Conjunctions involving objects 


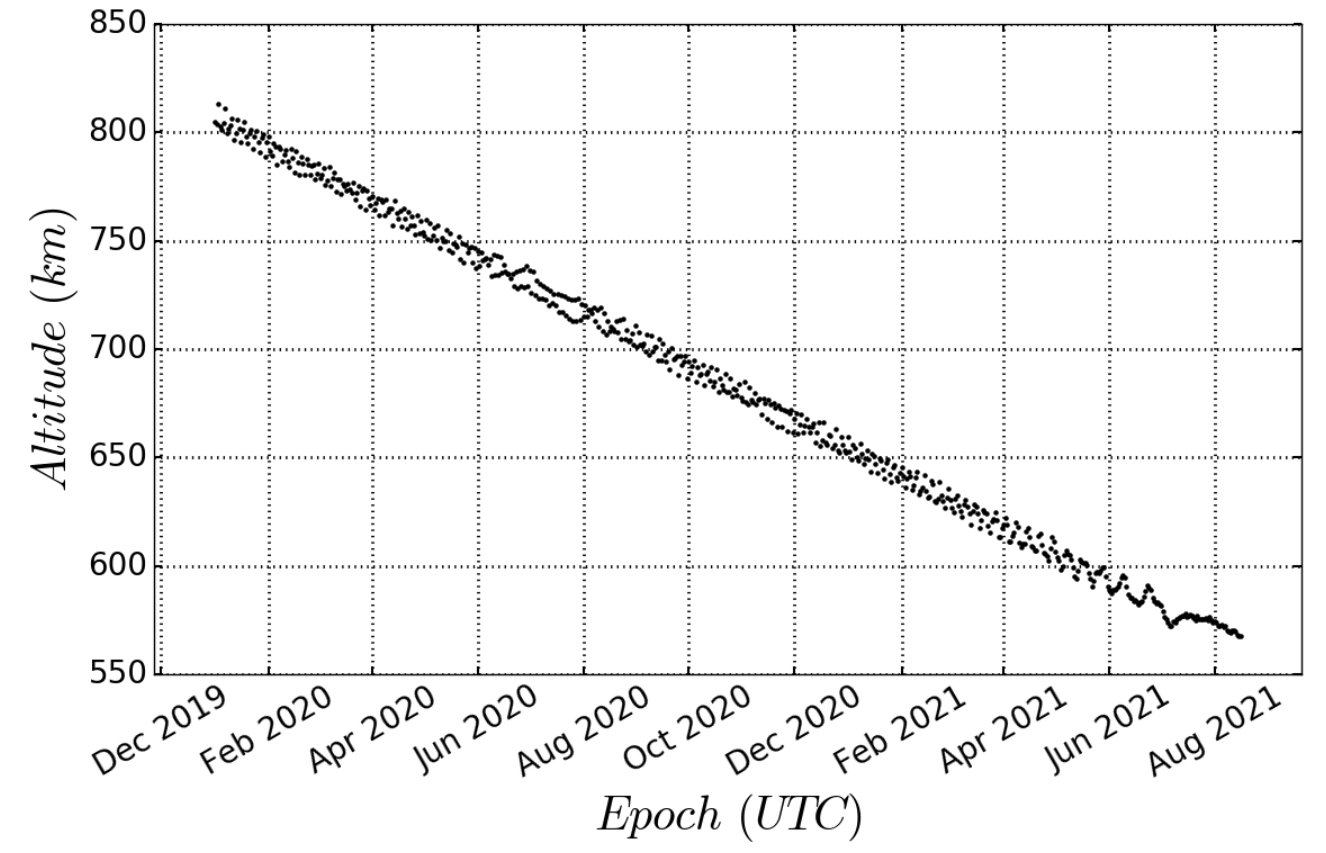

Figure 3: History of the altitude of the low-thrust trajectory lowering the altitude of Zenit-2. Assuming spherical Earth with radius of $6378.137 \mathrm{~km}$. 
that re-entered before the epoch of the conjunction were filtered out. Specific impulses of 300 and 3400 seconds (Larson \& Wetz, 1999) were used for chemical and low-thrust propulsion systems, respectively, in order to compute approximate fuel masses for the corresponding trajectories.

The reference accumulated collision probabilities for the targets were computed between 23 Oct 2013 and 23 Oct 2014, and 7 Nov 2014 and 7 Nov 2015 using the same ephemeris uncertainty, TLE set, objects' physical radii, and algorithms as for the examined trajectories. Such timing ensured that the least error was incurred by the SGP4 propagator because it was the closest to the epochs of most of the used TLEs. The collision probability is a function of ephemeris accuracy, which will be different for operational spacecraft carrying navigational instruments and derelicts, for example. The same ephemeris accuracy was used here for all the analysed cases, however, to provide a comparison between them. The key figures of merit for every trajectory, which are means of the values obtained with the two TLE catalogue snapshots, are given in Table 3 . Only the accumulated $P_{C}$, contribution of individual events and the number of conjunctions changed for every trajectory with different TLE catalogue snapshots. These data for every analysed snapshot are given in Table 4 . Table 5 provides averages per trajectory type, computed for every target and TLE snapshot epoch.

As can be anticipated, the longer a mission the higher the $P_{C}$ it tends to accumulate because more conjunctions take place (Anderson \& Schaub, 2015). On average, 6049 conjunctions took place for 269.21 day long trajectories (low-thrust) in Table 5, whereas 0.21 day long trajectories (impulsive no-drift) only experienced 12.0 conjunctions. These respective trajectories accumulated $P_{C}$ of $6.0 E-4$ and $3.7 E-16$. Mission duration would only not affect the accumulated $P_{C}$ if no conjunctions ever took place, which is unlikely if an object is to traverse the densely populated LEO regime in order to be removed from orbit. Generally, objects considered for ADR tend to reside in densely populated orbital regimes and so take part in conjunctions. This shows that leaving the targets in their unaltered orbits will cause them to keep accumulating collision probability beyond the one year reference values found in this study. However, the longer the ADR mission the lower the fuel mass and so the lower the cost (mass is proportional to launch cost (Larson \& Wetz, 1999)) - fuel masses of the low-thrust and impulsive no-drift trajectory types were 16.4 and $1477.3 \mathrm{~kg}$, respectively. This highlights that the cost and $P_{C}$ are conflicting drivers for ADR missions and so they should be traded off. Performing this trade-off study requires the relative importance 
of $P_{C}$ and cost to be agreed upon, however, which can only be done by the entities designing ADR missions.

The trend of $P_{C}$ being higher for longer missions can be offset by very close conjunctions. Conjunctions with low miss distance tend to have accordingly high collision probabilities. This is especially the case in certain relative geometries, where the uncertainty ellipsoids are aligned such that the combined covariance is relatively small. Figure 4, Fig. 5 and Fig. 6 illustrate this point. Three conjunctions gave rise to $97.5 \%$ of the $P_{C}$ that the low-thrust trajectory targeting Zenit-2 accumulated when screened against the 2013 TLE snapshot. For Tsyklon-3 and Kosmos-3M, single conjunctions gave rise to $99.9 \%$ and $99.0 \%$ of the final $P_{C}$ accumulated by their low-thrust trajectories, respectively. Similarly in the reference cases, the conjunctions with the highest $P_{C}$ contributed, on average, $56.4 \%, 43.1 \%$ and $30.1 \%$ to the final values accumulated by Zenit-2, Tsyklon-3 and Kosmos-3M, respectively. Table 3 shows that $P_{C}>1 E-4$ was observed for trajectories with contributions of at least $30.1 \%$ and durations of at least 121.24 days. Such a duration was sufficient for a relatively close, high- $P_{C}$ conjunction to take place for the investigated trajectories. However, a trajectory with any duration could involve a conjunction with a relatively high $P_{C}$, therefore this finding is not general.

The results show that the collision probability of every investigated ADR trajectory is the higher the longer the duration of the mission. Events that will have much higher collision probabilities than most others throughout the entire mission will occur, however. Such extremely dangerous conjunctions are normally avoided during spacecraft operations by postponing the manoeuvres or even conducting dedicated collision avoidance (Flohrer et al., 2009). Not every proposed ADR architecture provides such control, however. For example, low-thrust spacecraft have lower manoeuvrability and so they can only avoid collisions if those can be forecast well in advance (Anderson \& Schaub, 2015). However, conjunctions can only be predicted several days, or at most weeks, in advance due to the fact that uncertainty information stops reflecting reality the longer it is propagated (Vallado \& Seago, 2009). This could result in situations where a collision involving the object being removed would take place solely because its trajectory has been altered through ADR.

In fact, the collision probability of an object in an altered trajectory can be higher than if no ADR mission is flown to remove it for some time. Behaviour like this is not counter-intuitive but it was not observed in this study. Precautions ought to be made to avoid causing an orbital collision through 
Table 3: Mean figures of merit of the trajectories targeting Zenit-2, Tsyklon3 , and Kosmos-3M for the two TLE catalogue snapshots. Reference results for the targets left in their unaltered orbits for one year are also shown. The means were obtained using the data from Table 4.

\begin{tabular}{|c|c|c|c|c|c|c|c|}
\hline Target & 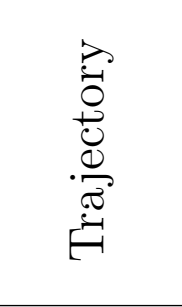 & 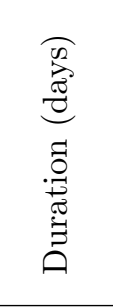 & $\begin{array}{l}\text { Tे } \\
\tilde{u} \\
\infty \\
\text { है } \\
\vec{\triangleleft}\end{array}$ & 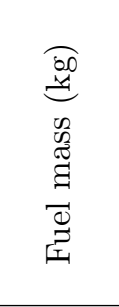 & 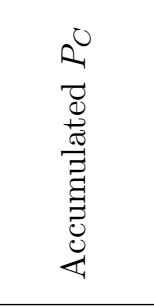 & 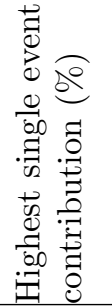 & 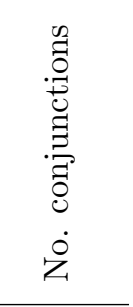 \\
\hline \multirow{4}{*}{ Zenit-2 } & Impulsive & 11.55 & 222.5 & 644.2 & $1.4 E-6$ & $99.9 \%$ & 384.5 \\
\hline & $\begin{array}{l}\text { Impulsive } \\
\text { no-drift }\end{array}$ & 0.13 & 751.1 & 1994.1 & $5.6 E-17$ & $42.9 \%$ & 23.5 \\
\hline & Low-thrust & 593.14 & 136.1 & 34.8 & $1.6 E-3$ & $8 \%$ & 15030.5 \\
\hline & Reference & 365.25 & $\mathrm{~N} / \mathrm{A}$ & $\mathrm{N} / \mathrm{A}$ & $1.3 E-3$ & $6.4 \%$ & 9564.5 \\
\hline \multirow{4}{*}{ Tsyklon-3 } & Impulsive & 11.62 & 173.5 & 80.1 & $9.9 E-13$ & $99.6 \%$ & 311.0 \\
\hline & $\begin{array}{l}\text { Impulsive } \\
\text { no-drift }\end{array}$ & 0.24 & 4927.9 & 1140.6 & $1.1 E-15$ & $6 \%$ & 7.5 \\
\hline & Low-thrust & 174.25 & 158.6 & 6.6 & $1.8 E-5$ & $90.9 \%$ & 1981.5 \\
\hline & Reference & 365.25 & $\mathrm{~N} / \mathrm{A}$ & $\mathrm{N} / \mathrm{A}$ & $2.3 E-4$ & $43.1 \%$ & 10797.0 \\
\hline \multirow{3}{*}{ Kosmos-3M } & Impulsive & 11.08 & 180.3 & 84.7 & $1.1 E-11$ & $99.9 \%$ & 233.0 \\
\hline & $\begin{array}{l}\text { Impulsive } \\
\text { no-drift }\end{array}$ & 0.26 & 6960.7 & 1297.3 & $0.0 E+0$ & $0.0 \%$ & 5.0 \\
\hline & $\begin{array}{l}\text { Low-thrust } \\
\text { Reference }\end{array}$ & $\begin{array}{l}121.24 \\
365.25\end{array}$ & $\begin{array}{r}179.4 \\
\text { N/A }\end{array}$ & $\begin{array}{r}7.6 \\
\mathrm{~N} / \mathrm{A}\end{array}$ & $\begin{array}{l}2.1 E-4 \\
4.9 E-4\end{array}$ & $\begin{array}{l}89.7 \% \\
30.1 \%\end{array}$ & $\begin{array}{l}1135.0 \\
9613.5\end{array}$ \\
\hline
\end{tabular}


Table 4: Collision probabilities, contributions of the events with the highest $P_{C}$ to the final accumulated value, and the numbers of conjunctions of the trajectories targeting Zenit-2, Tsyklon-3, and Kosmos-3M for the two TLE catalogue snapshots. Reference results for the targets left in their unaltered orbits for one year are also shown.

\begin{tabular}{|c|c|c|c|c|c|c|c|}
\hline \multirow[b]{2}{*}{ Target } & \multirow[b]{2}{*}{ 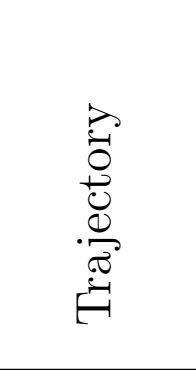 } & \multicolumn{3}{|c|}{23 Oct 2013} & \multicolumn{3}{|c|}{7 Nov 2014} \\
\hline & & 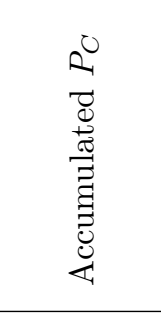 & 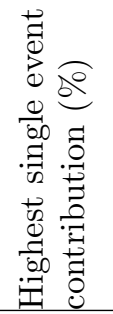 & 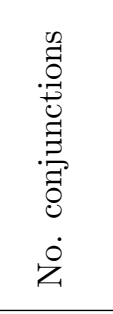 & 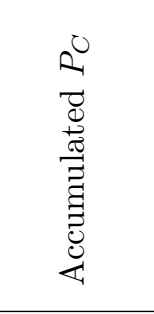 & 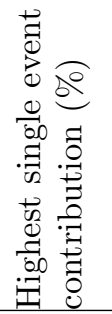 & 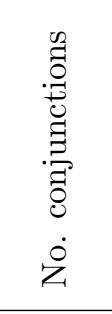 \\
\hline \multirow{4}{*}{ Zenit-2 } & Impulsive & $1.2 E-11$ & $99.9 \%$ & 379 & $2.7 E-6$ & $99.9 \%$ & 390 \\
\hline & $\begin{array}{l}\text { Impulsive } \\
\text { no-drift }\end{array}$ & $1.1 E-16$ & $85.9 \%$ & 23 & $0.0 E+0$ & $0.0 \%$ & 24 \\
\hline & Low-thrust & $1.6 E-3$ & $84.6 \%$ & 14580 & $1.6 E-3$ & $23.1 \%$ & 15481 \\
\hline & Reference & $1.9 E-3$ & $43.7 \%$ & 9645 & $8.3 E-4$ & $69.0 \%$ & 9484 \\
\hline \multirow{3}{*}{ Tsyklon-3 } & Impulsive & $2.1 E-15$ & $99.3 \%$ & 290 & $2.0 E-12$ & $99.9 \%$ & 332 \\
\hline & $\begin{array}{l}\text { Impulsive } \\
\text { no-drift }\end{array}$ & $2.1 E-15$ & $99.3 \%$ & 4 & $0.0 E+0$ & $0.0 \%$ & 11 \\
\hline & $\begin{array}{l}\text { Low-thrust } \\
\text { Reference }\end{array}$ & $\begin{array}{l}3.6 E-5 \\
2.1 E-4\end{array}$ & $\begin{array}{l}99.9 \% \\
27.1 \%\end{array}$ & $\begin{array}{r}1929 \\
10102\end{array}$ & $\begin{array}{l}9.3 E-8 \\
2.5 E-4\end{array}$ & $\begin{array}{l}82.0 \% \\
59.1 \%\end{array}$ & $\begin{array}{r}2034 \\
11492\end{array}$ \\
\hline \multirow{3}{*}{ Kosmos-3M } & Impulsive & $1.9 E-12$ & $99.9 \%$ & 228 & $2.1 E-11$ & $99.9 \%$ & 238 \\
\hline & $\begin{array}{l}\text { Impulsive } \\
\text { no-drift }\end{array}$ & $0.0 E+0$ & $0.0 \%$ & 5 & $0.0 E+0$ & $0.0 \%$ & 5 \\
\hline & $\begin{array}{l}\text { Low-thrust } \\
\text { Reference }\end{array}$ & $\begin{array}{l}4.1 E-4 \\
2.5 E-4\end{array}$ & $\begin{array}{l}99.0 \% \\
30.0 \%\end{array}$ & $\begin{array}{l}1169 \\
9422\end{array}$ & $\begin{array}{l}6.3 E-6 \\
7.4 E-4\end{array}$ & $\begin{array}{l}80.5 \% \\
30.2 \%\end{array}$ & $\begin{array}{l}1101 \\
9805\end{array}$ \\
\hline
\end{tabular}

Table 5: Average figures of merit for every investigated trajectory type, computed with the data for three targets and two TLE snapshots.

\begin{tabular}{l|rrrr}
\multicolumn{1}{c|}{ Trajectory type } & Impulsive & Impulsive no-drift & Low-thrust & Reference \\
\hline Duration (days) & 11.42 & 0.21 & 296.21 & 365.25 \\
$\Delta V\left(m\right.$ sec $\left.^{-1}\right)$ & 192.1 & 4213.2 & 156.5 & $\mathrm{~N} / \mathrm{A}$ \\
Fuel mass $(\mathrm{kg})$ & 269.7 & 1477.3 & 16.4 & $\mathrm{~N} / \mathrm{A}$ \\
Accumulated $P_{C}$ & $4.5 E-7$ & $3.7 E-16$ & $6.0 E-4$ & $6.9 E-4$ \\
No. conjunctions & 309.5 & 12.0 & 6049.0 & 9991.7
\end{tabular}



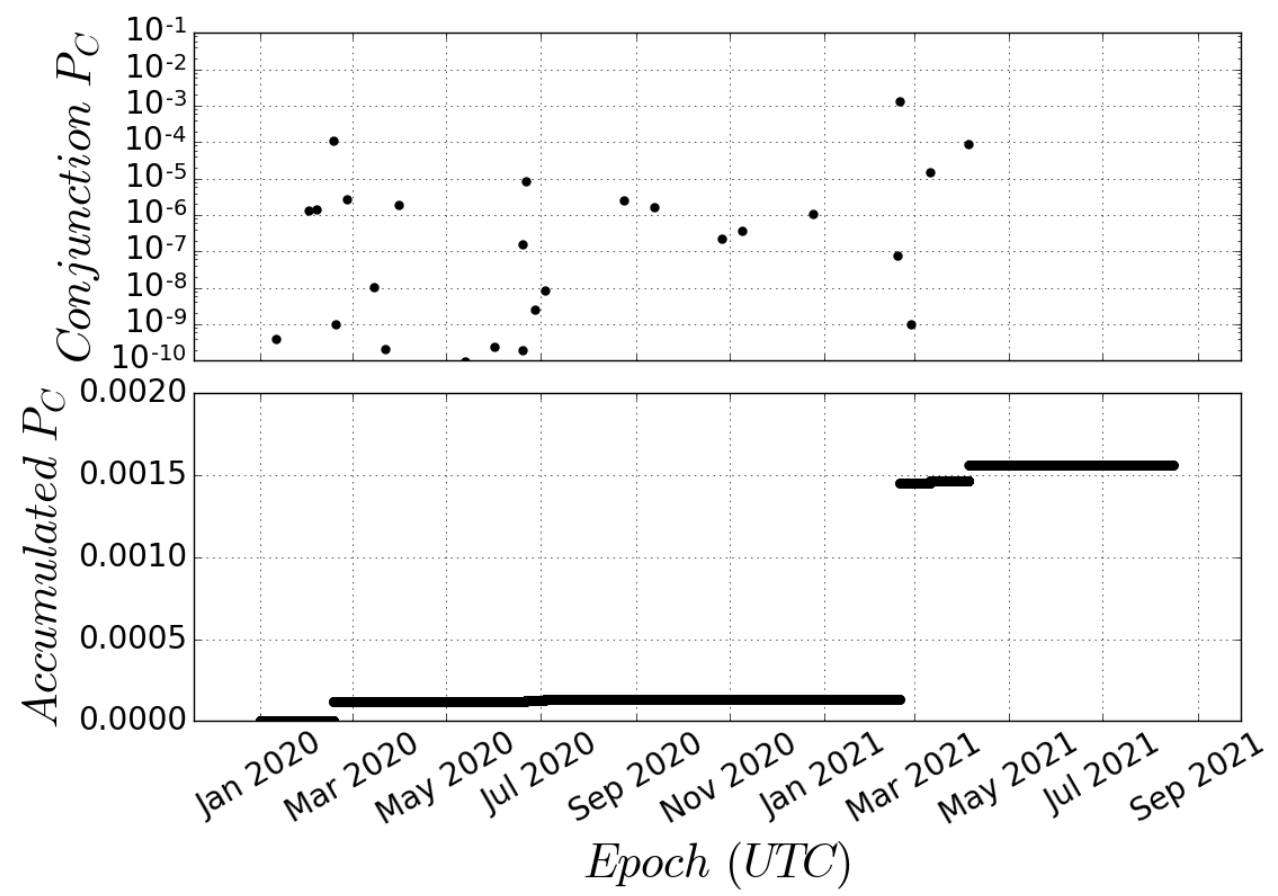

Figure 4: Collision probabilities of all the conjunctions of the low-thrust trajectory targeting Zenit-2 screened against the 2013 TLE snapshot, together with the evolution of the corresponding accumulated $P_{C}$. 

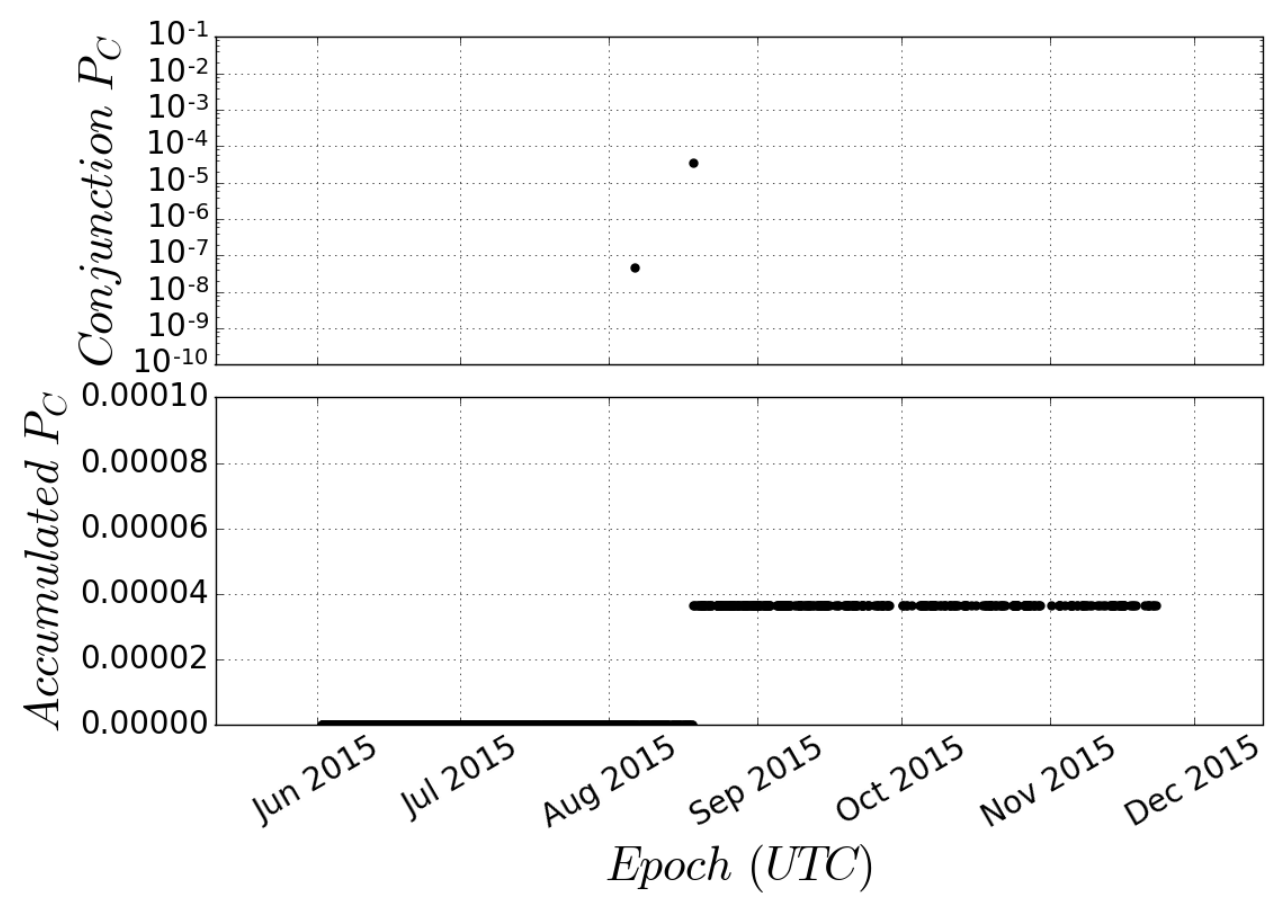

Figure 5: Collision probabilities of all the conjunctions of the low-thrust trajectory targeting Tsyklon-3 screened against the 2013 TLE snapshot, together with the evolution of the corresponding accumulated $P_{C}$. 

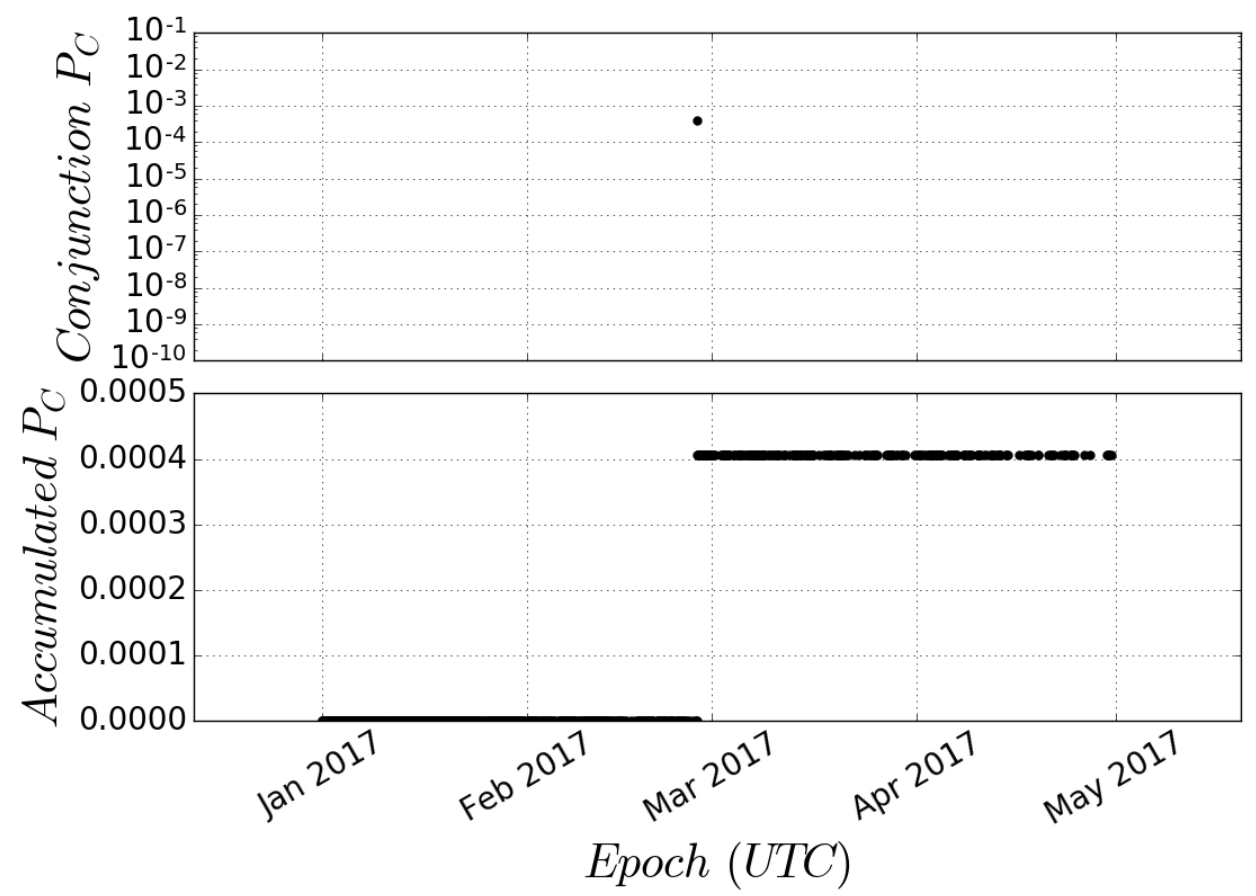

Figure 6: Collision probabilities of all the conjunctions of the low-thrust trajectory targeting Kosmos-3M screened against the 2013 TLE snapshot, together with the evolution of the corresponding accumulated $P_{C}$. 
ADR. This is not only because an event like this may negate the benefits of conducting ADR despite the considerable investments that will be needed. Causing a collision while performing ADR would likely cause debris owners and launching states to be reluctant to allow, let alone fund, further removal of their objects. This might also render the entity performing ADR liable because they would be in control of the object when it took part in the collision (Soucek, 2014).

The fact that an ADR mission may be more risky in the short-term than performing no debris remediation, however, should not be perceived as an argument against ADR. Leaving a large number of derelict, uncontrolled objects in orbit until their natural re-entry will cause these objects to accumulate arbitrarily high collision probabilities, which could result in collisions. It is the exact nature of the removal mission that is in question, not the very idea of performing ADR.

\section{Discussion}

Several simplifications were made when generating the data on which the findings of this work are based. This was done to reduce the computational time needed for the analysis and to make it feasible given the computational resources that were available. These simplifications include e.g. using approximate object sizes, ignoring their attitude, assuming uncertainty of the ephemerides, and making several simplifying assumptions when computing the collision probabilities of individual conjunctions. There are several ways in which these could have affected the collision probabilities of the investigated ADR trajectories.

Missing potentially very close conjunctions or incorrectly computing collision probabilities due to interpolation errors would alter the $P_{C}$ of individual trajectories. Some conjunctions could have been missed entirely, because they involved a classified object, which is not included in the public catalogue published through Space-Track (2013). Using a physical radius from the database for certain objects but not for the others could render the collision probabilities of the conjunctions involving the objects with assumed radius different to what they would be when using their actual size. On the other hand, using the database of sizes ensures that the objects much larger than others of the same type will give rise to higher collision probabilities as they would in reality. Furthermore, propagator errors, ignoring the attitude of the objects and other simplifying assumptions in the $P_{C}$ estimation algo- 
rithms mean that different collision probabilities of individual conjunctions could have been found had higher-fidelity algorithms been used.

However, the conjunctions provide a real-life example of a set of the possible events that could occur in reality, i.e. the outcome is plausible. Using the actual population of known objects on-orbit also ensured that the $P_{C}$ of every trajectory reflected the time it spent in densely populated orbital regimes.

Despite the assumptions and simplifications that were made, a large spectrum of collision probabilities was found for every object. For the low-thrust trajectory targeting Zenit-2 and screened against the 2013 TLE snapshot, the $P_{C}$ of individual conjunctions varied from less than $1.8 \times 10^{-308}$ (smallest double precision floating point number) to $1.34 \times 10^{-3}$. Collision probabilities of all the conjunctions were inversely proportional to the miss distance, scaled with size of the objects etc. as they would be when using the highest-fidelity methods and ephemerides. This means that the set of exemplar conjunction geometries found with the TLE catalogue snapshots provided a corresponding set of exemplar collision probabilities and the events with high collision probability were as infrequent as the ones with very low miss distance. Such high collision probability conjunctions do take place when using higher fidelity ephemerides and algorithms, which leads to conjunction screenings being routinely performed for operational spacecraft (Flohrer et al., 2009). The existence of such events is not an artefact resulting from the ephemerides or the assumed object sizes, for example.

It cannot be guaranteed that all the conjunctions of every trajectory were affected by the simplifications of this study in the same manner. This means that the contributions of the highest- $P_{C}$ events to the final collision probabilities may not be exactly what they would be if using the highest-fidelity input data and algorithms. However, this does not undermine the finding that the relatively infrequent, high- $P_{C}$ events dominate the others, which have lower collision probabilities, as far as final accumulated collision probability is concerned. Neither does this undermine the finding that certain ADR architectures will take more time but use less fuel to remove their targets. Such architectures will experience more conjunctions and thus accumulate larger $P_{C}$ than shorter ones. Because large fraction of $P_{C}$ stems from single events, however, the found relationship between trajectory duration and its $P_{C}$ could change if a conjunction with high $P_{C}$ took place for any trajectory.

The collision probabilities were computed using the cross-sectional area of only the target for all the investigated trajectories. In practice, every trajec- 
tory will be associated with some specific architecture, which will impact the cross-sectional area of the ADR spacecraft and so also its $P_{C}$. For example, long tethers or large solar arrays needed for electric propulsion will make such ADR missions even more risky than missions using chemical propulsion. If solar arrays or tethers are struck by debris, they will produce fragments that will have the potential to damage or disable active satellites. Even though such collisions are not likely to cause catastrophic breakups (Nock et al., 2013), they should be avoided because small debris are difficult to track and so the risk they pose to active spacecraft cannot be easily mitigated.

Lastly, only three exemplar ADR mission trajectory types and three targets were analysed. This sample of ADR trajectories was sufficient to demonstrate the mechanism that leads to accumulation of non-zero $P_{C}$ for such trajectories. In addition, high collision risk of an ADR vehicle tends to be associated with occurrence of single conjunctions with a relatively high $P_{C}$ (c.f. the rest of conjunctions) that contribute a large portion to the total collision probability. Similar behaviour was observed by Lidtke et al. (2015) when analysing all objects in the public catalogue. Specifically, for $27.8 \%$ of the objects, a single event contributed $10 \%$ or more to the $P_{C}$ accumulated over one month. Therefore, the impact of high- $P_{C}$ conjunctions found here is not an artefact that can be attributed to the relatively low number of analysed trajectories. Such high- $P_{C}$ events can take place for de-orbit trajectories of any duration, and thus the capacity to mitigate the associated risk becomes more important than reducing the de-orbiting time.

\section{Conclusions}

The potential of three different ADR trajectory types to cause orbital collisions whilst attempting to remove three example target objects was investigated. Even though this study focused on mission trajectories, these can be associated with specific groups of removal technologies.

It was noticed that longer ADR missions, which are cheaper because they use less fuel (16.4 instead of $1477.3 \mathrm{~kg}$ ), may be more prone to causing a collision $\left(P_{C}\right.$ of $\left.6.0 E-4 \mathrm{VS} 3.7 E-16\right)$ than other, more expensive solutions because of the longer de-orbiting time (269.21 instead of 0.21 days). This effect will be even more profound if an architecture-specific increase in the collision cross-sectional area is taken into account. It also shows that reducing the orbital lifetimes of derelicts will reduce the collision probabilities that they will accumulate. 
As much as $99 \%$ of the accumulated collision risk stems from relatively few, high- $P_{C}$ conjunctions. Even the very improbable conjunctions could cause collisions but our forecasting abilities limit the information available to support operational decisions for all spacecraft. Therefore, if extremely dangerous conjunctions are forecast, they should be avoided as they are our best estimates of the events that will cause collisions. Hence, ADR architectures that do not allow such avoidance actions and ignore the potential risk they introduce, should not be considered for implementation.

\section{Acknowledgements}

The authors would like to thank the ESA Space Debris office for allowing them to use the MASTER 2009 reference population, and Dr T.S. Kelso for provision of the objects' physical radii database.

Most of all, sincere thanks to Dr Claudio Bombardelli for providing an example low-thrust ADR trajectory.

The authors acknowledge the use of the IRIDIS High Performance Com-

puting Facility, and associated support services at the University of Southampton (UoS), as well as AGI's Systems Toolkit in the completion of this work.

Aleksander Lidtke was funded by an EPSRC Doctoral Training Grant awarded by the Faculty of Engineering and the Environment of UoS. Some of the reported work was undertaken as part of the European Commission FP7 LEOSWEEP project. The conjunctions found for the investigated ADR trajectories can be downloaded from the following link: http://dx.doi. org/10.5258/SOTON/396304.

\section{References}

Ash, R.L., O’Donoghue, P.J., Chambers, E.J., \& Raney, J.P., A methodology for selective removal of orbital debris, Adv. Space Res., 13(8), 243-247, 1993.

Alfano, S., Determining Satellite Close Approaches, Part II, The Journal of the Astronautical Sciences, 42, 143-152, 1994.

Alfano, S., Relating Position Uncertainty to Maximum Conjunction Probability, The Journal of the Astronautical Sciences, 53, 193-205, 2005. 
Alfano, S., Review of Conjunction Probability Methods for Short-term Encounters, AAS/AIAA Space Flight Mechanics Meeting, Sedona, AZ, USA, 2007.

Anderson, P.V., \% Schaub, H., Conjunction challenges of low-thrust geosynchronous debris removal maoeuvres, AAS/AIAA Space Flight Mechanics Meeting, Williamsburg, VA, USA, 2015.

Berend, N., Estimation of the probability of collision between two catalogued orbiting objects, Adv. Space Res., 23, 243-247, 1999.

Braun, V., Luepken, A., Flegel, S., Gelhaus, J., Moeckel, M., Kebschull, C., Wiedemann, C., \& Voersmann, P., Active debris removal of multiple priority targets, Adv. Space Res., 51, 1638-1648, 2013.

Budianto-Ho, I., Scalable Conjunction Processing Using Spatialotemporally Indexed Ephemeris Data, Advanced Maui Optical and Space Surveillance Technologies Conference, Wailea, HI, USA, 2014.

Cerf, M., Multiple Space Debris Collecting MissionDebris Selection and Trajectory Optimization, Journal of Optimization Theory and Applications, 156, 761-796, 2013.

Chamot, B., Richard, M., Salmon, T., Pisseloup, A., Cougnet, C., Axthelm, R., Saunders, C., Dupont, C., \& Lequette, L., Technology combination analysis tool (TCAT) for Active Debris Removal, 6th European Conference on Space Debris, Darmstadt, Germany, 2013.

Chan, K., International Space Station Collision Probability, The Aerospace Corporation, Chantilly, VA, USA, 2009.

Coppola, V., \& Woodburn, J., Determination of Close Approaches Based on Ellipsoidal Threat Volumes, Advances in the Astronautical Sciences, 102, 1013-1024, 1999.

ESA/ESOC Space Debris Office, Space Surveillance and Tracking System Requirements Document, Darmstadt, Germany, SST-SRD-4434, 2013.

Flohrer, T., Krag, H., \& Klinkrad, H., ESA's process for the identification and assessment of high-risk conjunction events, Adv. Space Res., 44, 355363, 2009. 
Frigm, R.C. \& Rohrbaugh, D., Relative Velocity as a Metric for Probability of Collision Calculations, Proceedings of the 59th International Astronautical Congress, Glasgow, Scotland, IAC-08-A6.2.5, 2008.

Furuta, S., Hanada, T., Fujita, K., \& Takezono, K., Discussion on the Necessity of Orbital Debris Removal in the Geostationary Region, 65th International Astronautical Congress, Toronto, Canada, IAC-14.A6.2.2, 2014.

Gaudel, A., Hourtolle, C., Goester, J.F., Fuentes, F., \& Ottaviani, M., DeOrbit Strategies With Low-Thrust Propulsion, Space Safety is No Accident, Springer International Publishing, 59-68, 2014.

Kessler, D.J., \& Cour-Palais, B.G., Collision frequency of artificial satellites: The creation of a debris belt, J. Geophys. Res., 83 (A6), 2637-2646, 1978.

Kim, I., Hirayama, H., \& Hanada, T., Practical guidelines for electrodynamic tethers to survive from orbital debris impacts, Adv. Space Res., 45(10), 1292-1300, 2010.

Krag, H., Klinkrad, H., Flohrer, T., Fletcher, E., \& Bobrinsky, N., The European Space Surveillance System - Required Performance and Design Concepts, 8th US/Russian Space Surveillance Workshop, Space Surveillance Detecting and Tracking Innovation, Maui, HI, USA, 2010.

Larson, W.J., \& Wertz, J.R., Space Mission Analysis and Design, Third Edition, Space Technology Library, El Segundo, CA, USA, 1999.

Lewis, H.G., Active Debris Removal: consequences of mission failure, 65th International Astronautical Congress, Toronto, Canada, IAC-14.A6.2.1, 2014 .

Lidtke, A.A.,\& Lewis, H.G., Collision probability assessment for active debris removal missions, 65th International Astronautical Congress, Toronto, Canada, IAC-12.A6.P.15, 2014.

Lidtke, A.A., Lewis, H.G., \& Armellin, R., Impact of high-risk conjunctions on Active Debris Removal target selection, Adv. Space Res., 56, (8) 17521764, 2015.

Martinez, S., Hourtolle, C., \& Goester, J.F., High Reactivity Maneuver Design in ATV Missions, 24th International Symposium on Space Flight Dynamics, Laurel, MD, USA, 2014. 
McKinley, D., Development of a Nonlinear Probability of Collision Tool, AIAA/AAS Astrodynamics Specialist Conference, Monterey, CA, USA, AIAA 02-4744, 2002.

McKnight, D.S., DiPentino, F., \& Knowles, S., Massive Collisions in LEO - A Catalyst to Initiate ADR, 65th International Astronautical Congress, Toronto, Canada, IAC-14.A6.2.1, 2014.

Nock, K.T., Aaron, K.M., \& McKnight, D.S., Removing Orbital Debris with Less Risk, Journal of Spacecraft and Rockets, 50, (2), 365-379, 2013.

Olympio, J. et al., Towards a better understanding of active debris removal options, Proceedings of the European Workshop on Active Debris Removal, Paris, France, 2010.

Pas, N., Lousada, J., Terhes, C., Bernabeu, M., \& Bauer, W., Target selection and comparison of mission design for space debris removal by DLR's advanced study group, Acta Astronautica, 102, 241-248, 2014.

Press, W.H., Flannery, B.P., Teukolsky, S.A., \& Vetterling, W.T., Numerical Recipes in $\mathrm{C}++, 2$ nd edition, Cambridge University Press, Cambridge, UK, 2002.

Reed, J., \& Barraclough, S., Development of a Harpoon System for Capturing Space Debris, 6th European Conference on Space Debris, Darmstadt, Germany, 2013.

Alarcon Rodriguez, J.R., Martinez Fadrique, F., \& Klinkrad, H., Collision risk assessment with a 'smart sieve' method, Joint ESA-NASA SpaceFlight Safety Conference, Noordwijk, the Netherlands, ESA SP-486, 2002.

Soucek, A., Active Debris Removal and questions of export control, 3rd European Workshop on Space Debris Modelling and Remediation, Paris, France, 2014.

Space-Track, Last accessed: 11 August 2014, URL: www.space-track.org, 2013.

Vallado, D., \& Seago, J.H., Covariance realism, AAS-AIAA Astrodynamics Specialist Conference, Pittsburgh, USA, 2009. 
Vance, L., \& Mense, A., Value analysis for orbital debris removal, Adv. Space Res., 52, 685-695, 2013.

Zenit stage 2, Russian Space Web, Last accessed: 17 December 2015, URL: www.russianspaceweb.com/zenit_stage2.html, 2013.

Tsyklon family, Russian Space Web, Last accessed: 17 December 2015, URL: www. russianspaceweb. com/tsyklon.html, 2015.

Zuiani, F., \& Vasile, M., Preliminary design of Debris removal missions by means of simplified Low-Thrust, many-revolution transfers, International Journal of Aerospace Engineering, 836250, 2012. 